\title{
A CRITERION FOR THE PARITY OF THE CLASS NUMBER OF AN ABELIAN FIELD WITH PRIME POWER CONDUCTOR
}

\author{
KEN-ICHI YOSHINO
}

\section{Introduction}

Let $f$ be a positive integer such that $f \not \equiv 2(\bmod 4)$. Let $h_{0}$ be the class number of the maximal real subfield of the $f$ th cyclotomic field $\mathbf{Q}\left(\zeta_{f}\right)$. It is interesting to determine when $h_{0}$ is even. Kummer [11] investigated this problem when $f$ is a prime and showed that if $h_{0}$ is even, then the relative class number $h^{*}$ of the cyclotomic field is even (Satz III). Moreover he gave another necessary condition for $h_{0}$ to be even (Satz IV). In [7] Hasse gave a necessary and sufficient condition for $h^{*}$ to be even (Satz 45). On the other hand G. Gras and M.-N. Gras [6] gave a criterion for the parity of the class number of a cyclic extension of $\mathbf{Q}$ of odd prime degree (Théorème III2, Corollaires III2 and III3). Moreover G. Gras [5] generalized the criterion for an abelian extension of $\mathbf{Q}$ of odd degree (Théorème III. 2 and Corollaire IV. 2). In this paper, by using Kummer's method in [11] and elementary argument, when $f$ is an odd prime power $p^{r}$, we shall simplify Théorème III. 2 in [5] and give a simple criterion for the parity of the class number of a real subfield of $\mathbf{Q}\left(\zeta_{f}\right)$. Our result is also related to Cornell and Rosen [3]. They showed that if $f$ is divisible by at least five primes, then $h_{0}$ is even and that if $f$ is divisible by exactly two, three or four primes, so is $h_{0}$ under certain condition respectively (Theorem A, Propositions 5 and 6). Their method in [3], however, does not yield anything when $f$ is a prime power. In section 1 we shall state our main results, i.e., Theorems 1 and 2 and their Corollaries. Among them, Theorem 1 is a simplification of Théorème III. 2 in [5] under the condition that $f$ is a prime power and takes a fundamental role to prove our criterion for the parity of the class number of a real subfield of $\mathbf{Q}\left(\zeta_{p^{r}}\right)$. In section 2 we shall prove Theorem 1 and Corollary by using four Lemmas. In section 3 we shall prove Theorem 2 and Corollary. In section 4 we shall give a few properties of invariants $\rho_{L}$ and $\mu_{L}$

Received May 2, 1995. 
defined in section 1 . In section 5 we shall give all the values of odd prime $p<3000$ such that the class number of the maximal real subfield of the $p$ th cyclotomic field is even (cf. [1], [13] p. 230).

The author would like to thank the referee for giving many helpful comments.

\section{Notations and result}

Let $p$ be an odd prime and $r$ a positive integer. Let $g$ be a primitive root modulo $p^{r}$ and $g_{i}$ the least positive residue of $g^{r}$ modulo $p^{r}$ for every $i \in \mathbf{Z}$. Then $g_{i+\varphi\left(p^{r}\right)}=g_{i}$ for every $i \in \mathbf{Z}$, where $\varphi$ is the Euler totient function. Let $\zeta=\zeta_{p^{r}}=$ $\cos \left(2 \pi / p^{r}\right)+\sqrt{-1} \sin \left(2 \pi / p^{r}\right)$. This is a primitive $p^{r}$ th root of unity. For every $i \in \mathbf{Z}$, we put

$$
\varepsilon_{i}=\frac{\zeta^{g_{i+1}}-\zeta^{-g_{i+1}}}{\zeta^{g_{i}}-\zeta^{-g_{i}}}=\frac{\sin \frac{2 g_{i+1} \pi}{p^{r}}}{\sin \frac{2 g_{i} \pi}{p^{r}}},
$$

which is called a cyclotomic unit of $\mathbf{Q}\left(\zeta+\zeta^{-1}\right)$. Putting $n=\varphi\left(p^{r}\right) / 2$, we have $\varepsilon_{n+i}=\varepsilon_{i}$ for each $i \in \mathbf{Z}$ and $\varepsilon_{0} \varepsilon_{1} \cdots \varepsilon_{n-1}=-1$. Let $E_{0}$ be the group of units of $\mathbf{Q}\left(\zeta+\zeta^{-1}\right)$ and $E_{C}$ the subgroup of $E_{0}$ generated by cyclotomic units, i.e. $E_{C}=$ $\left\langle\varepsilon_{0}, \varepsilon_{1}, \ldots, \varepsilon_{n-1}\right\rangle$. Let $h_{0}$ be the class number of $\mathbf{Q}\left(\zeta+\zeta^{-1}\right)$. Then it is well known that $h_{0}=\left[E_{0}: E_{C}\right]$ (cf. [7]). For every $i \in \mathbf{Z}$, we let $c_{\imath}=0$ or 1 according as $\varepsilon_{i}$ is positive or negative. We note that $2 g_{i}-2 g_{i+1}=g_{i+s}-g_{i+1+s} \pm p^{r} c_{i}$ and therefore that $c_{i} \equiv g_{i+s}-g_{i+1+s}(\bmod 2)$, where $s$ is the integer such that $g_{s}=2$, $1 \leq s<\varphi\left(p^{r}\right)$. (cf. [11]).

Let $L$ be a real subfield of $\mathbf{Q}(\zeta)$ and $m$ the degree of $L$. We denote by $E_{L}$ the group of units of $L$ and by $E_{C_{L}}$ the subgroup of $E_{L}$ generated by the cyclotomic units of $L$, i.e., $E_{c_{L}}=\left\langle\eta_{0}, \eta_{1}, \ldots, \eta_{m-1}\right\rangle$, where $\eta_{i}=N_{\mathbf{Q}^{\left(\zeta+\zeta^{-1}\right) / L}}\left(\varepsilon_{\imath}\right)$ for every $i \in$ Z. Then the class number $h_{L}$ of $L$ is represented by $h_{L}=\left[E_{L}: E_{C_{L}}\right]$. We let $d_{\imath}=$ 0 or 1 by

$$
d_{i} \equiv \sum_{j=0}^{\frac{n}{m}-1} c_{i+m j} \quad(\bmod 2)
$$

for every $i \in \mathbf{Z}$. We note that $d_{i}=0$ or 1 according as $\eta_{i}$ is positive or negative and that $d_{i+m}=d_{i}$ for every $i \in \mathbf{Z}$. We then define the $m$ by $m$ matrices

$$
M_{L}=\left(d_{i+j}\right)_{0 \leq i, j<m} \text { and } M_{L}^{*}=\left(d_{i-\jmath}\right)_{0 \leq i, j<m} .
$$

These matrices $M_{L}$ and $M_{L}^{*}$ are concerned with the Demjanenko matrix (cf. [8], 
[12]). Using these matrices $M_{L}$ and $M_{L}^{*}$, we give a criterion for the parity of the class number of $L$. Let $\mathbf{F}_{2}=\mathbf{Z} / 2 \mathbf{Z}$. For any matrix $M$ with coefficients in $\mathbf{Z}$, let $\operatorname{rank}_{\mathbf{F}_{2}} M$ denote the $\mathbf{F}_{2}$-rank of $M$, namely, the rank of the reduction of $M$ modulo 2. Let $\rho_{L}$ and $\mu_{L}$ be the $\mathbf{F}_{2}$-defects of $M_{L}$ and $\left(\begin{array}{c}M_{L} \\ M_{L}^{*}\end{array}\right)$, respectively. That is, we let

$$
\rho_{L}=m-\operatorname{rank}_{\mathbf{F}_{2}} M_{L}, \quad \mu_{L}=m-\operatorname{rank}_{\mathbf{F}_{2}}\left(\begin{array}{c}
M_{L} \\
M_{L}^{*}
\end{array}\right) \text {. }
$$

Then $0 \leq \mu_{L} \leq \rho_{L} \leq m$.

Now we denote by $E_{C_{L}}^{+}$the group of totally positive units in $E_{C_{L}}$. Let $E_{U_{L}}$ be the group of primary units in $E_{C_{L}}$, i.e., $E_{U_{L}}=\left\{\eta \in E_{C_{L}} ; \alpha^{2} \equiv \eta(\bmod 4)\right.$ for some integer $\alpha \in L\}$ (cf. [9] $\$ 59, \S 61$ ). Let $\sigma$ be the generator of the Galois group of $\mathbf{Q}(\zeta)$ over $\mathbf{Q}$ such that $\zeta^{\sigma}=\zeta^{g}$. The aim of this paper is to prove the following theorems and corollaries.

Theorem 1. Let $p$ be an odd prime. Let $L$ be a real abelian field of degree $m$ with conductor $p^{r}(r \geq 1)$. Let $x_{0}, x_{1}, \ldots, x_{m-1}$ be rational integers. Then $\eta_{0}^{x_{0}} \eta_{1}^{x_{1}} \ldots$ $\eta_{m-1}^{x_{m-1}} \in E_{U_{L}}$ if and only if $\eta_{0}^{x_{0}} \eta_{1}^{x_{m-1}} \eta_{2}^{x_{m-2}} \cdots \eta_{m-1}^{x_{1}} \in E_{C_{L}}^{+}$. Therefore $E_{U_{L}}$ is characterized by $E_{U_{L}}=\left\{\eta_{0}^{x_{0}} \eta_{1}^{x_{1}} \cdots \eta_{m-1}^{x_{m-1}} \in E_{C_{L}} ; M_{L}^{*} \boldsymbol{x} \equiv \mathbf{o}(\bmod 2)\right\}$, where $\boldsymbol{x}={ }^{t}\left(x_{0}, x_{1}, \ldots\right.$, $\left.x_{m-1}\right)$ is the transpose of $\left(x_{0}, x_{1}, \ldots, x_{m-1}\right)$ and $\mathbf{0}$ is the zero vector of size $m$.

Remark 1. Theorem 1 is a simplification of Théorème III. 2 in [5]. In fact, since $\eta_{i}=\eta_{0}^{\sigma^{i}}$ for every $i \in \mathbf{Z}$, we have $E_{C_{L}}=\eta_{0}^{\mathbf{Z}[\sigma]}$. We consider the automorphism of $\mathbf{Z}[\sigma]$ induced by $\sigma \rightarrow \sigma^{-1}$. By the automorphism, each element $\eta_{0}^{x_{0}} \eta_{1}^{x_{1}} \cdots$ $\eta_{m-1}^{x_{m-1}}=\eta_{0}^{x_{0}+x_{1} \sigma+\cdots+x_{m-1} \sigma^{m-1}}$ of $E_{U_{L}}$ is corresponding to $\eta_{0}^{x_{0}+x_{1} \sigma^{-1}+\cdots+x_{m-1} \sigma^{-(m-1)}}=$ $\eta_{0}^{x_{0}} \eta_{1}^{x_{m-1}} \cdots \eta_{m-1}^{x_{1}}$ of $E_{C_{L}}^{+}$

Our criterion for the parity of the class number is as follows.

COROLlary. Let $p$ be an odd prime. Let $L$ be a real abelian field with conductor $p^{\mathrm{r}}(r \geq 1)$ and $h_{L}$ the class number of $L$. Then $h_{L}$ is even if and only if $\mu_{L}>0$.

THEOREM 2. Let $p$ be an odd prime and $r$ a positive integer. Let $K$ be an imaginary abelian field with conductor $p^{r}$. Let $K_{0}$ be the maximal real subfield of $K$ and $h_{K}^{*}$ the relative class number of $K$. Then $h_{K}^{*} \equiv \operatorname{det} M_{K_{0}}(\bmod 2)$.

COROLlary. For an imaginary abelian field $K$ with conductor $p^{r}, h_{K}^{*}$ is even if and only if $\rho_{K_{0}}>0$. 
Remark 2. For an imaginary subfield $K$ of $\mathbf{Q}\left(\zeta_{p^{r}}\right)$, it follows from the above two Corollaries that if $h_{K_{0}}$ is even, then $h_{K}^{*}$ is even, since $\rho_{K_{0}} \geq \mu_{K_{0}} \geq 0$.

\section{Proof of Theorem 1 and Corollary}

Let $p$ be an odd prime. Let $L$ be a real subfield of $\mathbf{Q}(\zeta)$ not contained in $\mathbf{Q}\left(\zeta^{p}\right)$, where $\zeta=\zeta_{p^{r}}$. Let $h_{L}$ be the class number of $L, m$ the degree of $L$ and $n=\varphi\left(p^{r}\right) / 2$. To prove Theorem 1, we need the following three lemmas. From now on, for the sake of simplicity of notations, we put $E_{C}=E_{C_{L}}, E_{C}^{+}=E_{C_{L}}^{+}$and $E_{U}=E_{U_{L}}$.

Lemma 1. Let $\eta=\eta_{0}^{x_{0}} \eta_{1}^{x_{1}} \cdots \eta_{m-1}^{x_{m-1}}$ be a unit of $E_{C}$. Then $\eta \in E_{C}^{+}$if and only if $M_{L} x \equiv \mathbf{o}(\bmod 2)$, where $x={ }^{t}\left(x_{0}, x_{1}, \cdots, x_{m-1}\right)$ and $\mathbf{0}$ is the zero vector of size m. Therefore \# $E_{C}^{+} / E_{C}^{2}=2^{\rho_{L}}$.

Proof. It is obvious from the definition of $M_{L}$ and $\rho_{L}$.

Lemma 2. Let $s$ be the integer such that $g_{s}=2,1 \leq s<\varphi\left(p^{r}\right)$. Then $E_{U}=$ $\left\{\eta \in E_{C} ; \eta^{2} \equiv \eta^{\sigma^{s}}(\bmod 4)\right\}$.

Proof. Suppose that $\eta \in E_{U}$. Then there is an integer $\alpha$ such that $\alpha^{2} \equiv \eta$ $(\bmod 4)$. Here $\alpha$ is written as $\alpha=\sum_{i=0}^{\varphi\left(p^{r}\right)-1} x_{i} \zeta^{2}\left(x_{i} \in \mathbf{Z}\right)$. So we have $\alpha^{2} \equiv$ $\sum_{i=0}^{\varphi\left(p^{r}\right)-1} x_{i} \zeta^{2 i}=\alpha^{\sigma^{s}}(\bmod 2)$. Hence $\alpha^{4} \equiv \alpha^{2 \sigma^{s}}(\bmod 4)$. Therefore $\eta^{2} \equiv \alpha^{4} \equiv \alpha^{2 \sigma^{s}}$ $\equiv \eta^{\sigma^{s}}(\bmod 4)$. This completes the proof.

Lemma 3. $\sum_{b=1}^{p^{r}-1} \frac{\zeta_{p^{r}}^{-b g_{u}}}{1-\zeta_{p^{r}}^{b}}=\frac{p^{r}-1}{2}-g_{u} \quad$ for every $u \in \mathbf{Z}$

Proof. Let $\zeta=\zeta_{p^{r}}$ and $S=\sum_{b=1}^{p^{r}-1} \frac{\zeta^{-b g_{u}}}{1-\zeta^{b}}$. Then we note that $S$ is a real number. Putting $a=g_{u}$, we get

$$
\begin{aligned}
S & =\sum_{b=1}^{p^{r}-1} \frac{\zeta^{-a b}}{1-\zeta^{b}}=\sum_{b=1}^{p^{r}-1} \frac{1}{\zeta^{(a-1) b}}\left\{\frac{1}{\zeta^{b}}+\frac{1}{1-\zeta^{b}}\right\} \\
& =\sum_{b=1}^{p^{r}-1} \frac{1}{\zeta^{(a-2) b}}\left\{\frac{1}{\zeta^{2 b}}+\frac{1}{\zeta^{b}}+\frac{1}{1-\zeta^{b}}\right\} \\
& =\sum_{b=1}^{p^{r}-1}\left\{\frac{1}{\zeta^{a b}}+\frac{1}{\zeta^{(a-1) b}}+\cdots+\frac{1}{\zeta^{2 b}}+\frac{1}{\zeta^{b}}+\frac{1}{1-\zeta^{b}}\right\}
\end{aligned}
$$




$$
=-a+\frac{p^{r}-1}{2}
$$

Thus we obtain the desired equation.

Proof of Theorem 1. We note that it suffices to show the equivalence in the case $L=\mathbf{Q}\left(\zeta+\zeta^{-1}\right)$. In fact, since $\eta_{\imath}=N_{\mathbf{Q}\left(\zeta+\zeta^{-1) / L}\right.}\left(\varepsilon_{i}\right)$ for each $i \in \mathbf{Z}$, we have $\eta_{0}^{x_{0}} \eta_{1}^{x_{1}} \cdots \eta_{m-1}^{x_{m-1}}=\Pi_{i=0}^{m-1} \Pi_{j=0}^{\frac{n}{m}-1} \varepsilon_{i+m j}^{x_{i}}=\Pi_{i=0}^{m-1} \Pi_{j=0}^{\frac{n}{m}-1} \varepsilon_{i+m j}^{x_{i+m j}}$ and $\eta_{0}^{x_{0}} \eta_{1}^{x_{m-1}} \cdots \eta_{m-1}^{x_{1}}=$ $\Pi_{i=0}^{m-1} \Pi_{j=0}^{\frac{n}{m}-1} \varepsilon_{\imath+m j}^{x_{m-t}}=\Pi_{i=0}^{m-1} \Pi_{j=0}^{\frac{n}{m}-1} \varepsilon_{i+m j}^{x_{n-i-m s}}$, where $x_{i+m j}=x_{i}$ for any $i, j \in \mathbf{Z}$. In the case $f=p(r=1)$, Kummer essentially showed that if $\varepsilon_{0}^{x_{0}} \varepsilon_{1}^{x_{1}} \cdots \varepsilon_{n-1}^{x_{n-1}} \in E_{U}$, then $\varepsilon_{0}^{x_{0}} \varepsilon_{1}^{x_{n-1}} \varepsilon_{2}^{x_{n-2}} \cdots \varepsilon_{n-1}^{x_{1}} \in E_{C}^{+}$([11] p. 866 p. 868). Now, in order to prove the converse in the case $f=p$, we summarize his proof as follows: Let $s$ be the integer such that $g_{s}=2,1 \leq s<\varphi(p)$. Let $\alpha$ be the number of $\mathbf{Q}\left(\zeta+\zeta^{-1}\right)$ such that $\varepsilon_{0}^{2}$ $-\varepsilon_{0}^{\sigma^{s}}=2 \alpha \varepsilon_{0}^{\sigma^{s}}$. Then

$$
\alpha \equiv \frac{-1}{1-\zeta^{2}}+\frac{1}{1-\zeta^{2 g}}(\bmod 2),
$$

where both numbers are 2 -integral. Putting $\varepsilon=\varepsilon_{0}^{x_{0}} \varepsilon_{1}^{x_{1}} \varepsilon_{2}^{x_{2}} \cdots \varepsilon_{n-1}^{x_{n-1}}$, we get

$$
\varepsilon^{2} \equiv \varepsilon^{\sigma^{s}}\left\{1+2 \sum_{i=0}^{n-1} x_{\imath} \alpha^{\sigma^{i}}\right\}(\bmod 4)
$$

Hence, by Lemma $2, \varepsilon \in E_{U}$ if and only if $\sum_{t=0}^{n-1} x_{\imath} \alpha^{\sigma^{i}} \equiv 0(\bmod 2)$. The latter condition is equivalent to

$$
\sum_{i=0}^{n-1} x_{i}\left(\frac{-1}{1-\zeta^{2 g^{i}}}+\frac{1}{1-\zeta^{2 g^{i+1}}}\right) \equiv 0 \quad(\bmod 2) .
$$

Here we replace $\zeta$ by $\zeta^{a}$. Multiplying $\zeta^{-2 a g^{j+1}}$ in both sides, summing them with respect to $a=1,2, \cdots, p-1$ and using Lemma $3(r=1)$, i.e.,

$$
\sum_{b=1}^{p-1} \frac{\zeta^{-b g^{i}}}{1-\zeta^{b}}=\frac{p-1}{2}-g_{i},
$$

we have $\sum_{i=0}^{n-1} x_{i}\left(g_{j+1-i}-g_{j-\imath}\right) \equiv 0(\bmod 2)$ for every $j$. Since $c_{i} \equiv g_{i+s}-g_{i+1+s}$ $(\bmod 2)$, we obtain $\sum_{t=0}^{n-1} x_{i} c_{k-i} \equiv 0(\bmod 2)$ for every $k$. This implies that $\varepsilon_{0}^{x_{0}} \varepsilon_{1}^{x_{n-1}} \varepsilon_{2}^{x_{n-2}} \cdots \varepsilon_{n-1}^{x_{1}} \in E_{C}^{+}$by Lemma 1 .

Now we consider the converse in the case $f=p$. By above argument it suffices to show that $\sum_{i=0}^{n-1} x_{i}\left(g_{j+1-i}-g_{j-i}\right) \equiv 0(\bmod 2)$ for every $j$ implies 


$$
\sum_{i=0}^{n-1} x_{i}\left(\frac{-1}{1-\zeta^{2 g^{i}}}+\frac{1}{1-\zeta^{2 g^{i+1}}}\right) \equiv 0(\bmod 2) .
$$

This is proved as follows. Indeed,

$$
\begin{aligned}
p \sum_{i=0}^{n-1} x_{i}\left(\frac{-1}{1-\zeta^{2 g^{i}}}+\frac{1}{1-\zeta^{2 g^{i+1}}}\right) & =\sum_{i=0}^{n-1} x_{i} \sum_{k=1}^{p-1} k\left(\zeta^{2 g^{i} k}-\zeta^{2 g^{i+1} k}\right) \\
& =\sum_{i=0}^{n-1} x_{i} \sum_{u=0}^{p-2} g_{u}\left(\zeta^{2 g^{i+u}}-\zeta^{2 g^{i+u+1}}\right) \\
& =\sum_{i=0}^{n-1} x_{i} \sum_{u=0}^{p-2}\left(g_{u}-g_{u-1}\right) \zeta^{2 g^{i+u}}
\end{aligned}
$$

Here we replace the lattice point $(i, u)$ in the region $0 \leq i, u$ and $i+u<n-1$ by the point $(i, u+2 n)$ and put $k=i+u$, where $2 n=p-1$. Then, since $g_{u+p-1}=g_{u}$, we have

$$
\sum_{i=0}^{n-1} x_{i}\left(\frac{-1}{1-\zeta^{2 g^{i}}}+\frac{1}{1-\zeta^{2 g^{i+1}}}\right) \equiv \sum_{k=n-1}^{3 n-2} \zeta^{2 g^{k}} \sum_{i=0}^{n-1} x_{i}\left(g_{k-i}-g_{k-i-1}\right) \equiv 0(\bmod 2) .
$$

Thus Theorem 1 is proved in the case $f=p$.

Next, by induction, we shall prove Theorem 1 in the case $f=p^{r}(r>1)$. That is, we assume that the assertion in Theorem 1 is true in the case $f=p^{r-1}$ and prove that it holds true in the case $f=p^{r}$. Let $N$ be the norm from $\mathbf{Q}\left(\zeta_{p^{r}}+\right.$ $\left.\zeta_{p^{r}}^{-1}\right)$ to $\mathbf{Q}\left(\zeta_{p^{r-1}}+\zeta_{p^{r-1}}^{-1}\right)$ and let $n=\varphi\left(p^{r}\right) / 2, m=\varphi\left(p^{r-1}\right) / 2$. We denote by $E_{C}^{+(r)}$ and $E_{U}^{(r)}$ the groups $E_{C}^{+}$and $E_{U}$ for $L=\mathbf{Q}\left(\zeta_{p^{r}}+\zeta_{p^{r}}^{-1}\right)$, respectively. Similarly we use the notation $c_{\imath}^{(r)}$ and $g_{\imath}^{(r)}$ for $f=p^{r}$. Let $\zeta=\zeta_{p^{r}}$ and $\zeta_{0}=\zeta_{p^{r-1}}$. Then $N\left(E_{C}^{+(r)}\right) \subseteq E_{C}^{+(r-1)}$ and $N\left(E_{U}^{(r)}\right) \subseteq E_{U}^{(r-1)}$.

Now suppose that $\varepsilon=\varepsilon_{0}^{x_{0}} \varepsilon_{1}^{x_{1}} \cdots \varepsilon_{n-1}^{x_{n-1}} \in E_{U}^{(r)}$. This implies that

$$
\sum_{i=0}^{n-1} x_{\imath}\left(\frac{-1}{1-\zeta^{2 g^{i}}}+\frac{1}{1-\zeta^{2 g^{i+1}}}\right) \equiv 0(\bmod 2) \text {. }
$$

We shall show that $\varepsilon_{0}^{x_{0}} \varepsilon_{1}^{x_{n-1}} \cdots \varepsilon_{n-1}^{x_{1}} \in E_{C}^{+(r)}$. By assumption we have $N(\varepsilon)=$ $N\left(\varepsilon_{0}^{x_{0}} \varepsilon_{1}^{x_{1}} \cdots \varepsilon_{n-1}^{x_{n-1}}\right)=\eta_{0}^{y_{0}} \eta_{1}^{y_{1}} \cdots \eta_{m-1}^{y_{m-1}} \in E_{U}^{(r-1)}$, where $\eta_{i}=N\left(\varepsilon_{i}\right)$ and $y_{i}=\sum_{j=0}^{\frac{n}{m}-1}$ $x_{i+j m}$ for every $i$. Multiplying $\zeta^{-2 g^{j+1}}$ in both sides of the above congruence, replacing $\zeta$ by $\zeta^{a}$ and summing them with respect to $a \in\left\{1,2, \ldots, p^{r}-1\right\}$ prime to $p$, we obtain

$$
\sum_{i=0}^{n-1} x_{i} \sum_{\substack{a=1 \\(a, p)=1}}^{p^{r}-1}\left(\frac{-\zeta^{-2 a g^{j+1}}}{1-\zeta^{2 a g^{i}}}+\frac{\zeta^{-2 a g^{j+1}}}{1-\zeta^{2 a g^{i+1}}}\right) \equiv 0(\bmod 2) .
$$

Hence 


$$
\sum_{i=0}^{n-1} x_{i} \sum_{\substack{b=1 \\(b, p)=1}}^{p^{r}-1}\left(\frac{-\zeta^{b g^{j+1-i}}}{1-\zeta^{b}}+\frac{\zeta^{-b g^{j-i}}}{1-\zeta^{b}}\right) \equiv 0(\bmod 2) .
$$

Here we divide the left side into two parts, i.e.,

$$
\sum_{i=0}^{n-1} x_{i} \sum_{b=1}^{p^{r}-1}\left(\frac{-\zeta^{b g^{j+1-i}}}{1-\zeta^{b}}+\frac{\zeta^{-b g^{j-i}}}{1-\zeta^{b}}\right)-\sum_{i=0}^{n-1} x_{i} \sum_{b=1}^{p^{r-1}-1}\left(\frac{-\zeta_{0}^{b g^{j+1-i}}}{1-\zeta_{0}^{b}}+\frac{\zeta_{0}^{-b g^{j-i}}}{1-\zeta_{0}^{b}}\right) \equiv 0(\bmod 2) .
$$

Therefore it follows from Lemma 3 that

$$
\sum_{i=0}^{n-1} x_{i}\left(g_{j+1-i}^{(r)}-g_{j-i}^{(r)}\right)-\sum_{i=0}^{n-1} x_{i}\left(g_{j+1-i}^{(r-1)}-g_{j-i}^{(r-1)}\right) \equiv 0(\bmod 2) .
$$

Let $s=s^{(r)}$ and $s_{0}=s^{(r-1)}$ be the integers such that $g_{s}^{(r)}=g_{s_{0}}^{(r-1)}=2,1 \leq s$ $<\varphi\left(p^{r}\right)$ and $1 \leq s_{0}<\varphi\left(p^{r-1}\right)$. Thus

$$
\sum_{i=0}^{n-1} x_{i} c_{j-i-s}^{(r)}-\sum_{i=0}^{n-1} x_{i} c_{j-i-s_{0}}^{(r-1)} \equiv 0(\bmod 2)
$$

since $c_{i}^{(r)} \equiv g_{i+s}^{(r)}-g_{i+1+s}^{(r)}(\bmod 2)$ for every $i$. By the assumption of induction, $\eta_{0}^{y_{0}} \eta_{1}^{y_{1}} \cdots \eta_{m-1}^{y_{m-1}} \in E_{U}^{(r-1)}$ implies $\eta_{0}^{y_{0}} \eta_{1}^{y_{m-1}} \cdots \eta_{m-1}^{y_{1}} \in E_{C}^{+(r-1)}$, i.e., $\sum_{i=0}^{m-1} y_{\imath} c_{k-\imath}^{(r-1)} \equiv 0$ $(\bmod 2)$ for every $k$. Therefore

$$
\sum_{i=0}^{n-1} x_{i} c_{j-i-s_{0}}^{(r-1)}=\sum_{i=0}^{m-1} \sum_{k=0}^{\frac{n}{m}-1} x_{i+m k} c_{j-i-m k-s_{0}}^{(r-1)}=\sum_{i=0}^{m-1} y_{i} c_{j-i-s_{0}}^{(r-1)} \equiv 0(\bmod 2) .
$$

Here we note that $c_{j-\imath-m k-s_{0}}^{(r-1)}=c_{j-\imath-s_{0}}^{(r-1)}$. Thus we have $\sum_{l=0}^{n-1} x_{i} c_{k-i}^{(r)} \equiv 0(\bmod 2)$ for every $k$, which shows that $\varepsilon_{0}^{x_{0}} \varepsilon_{1}^{x_{n-1}} \cdots \varepsilon_{n-1}^{x_{1}} \in E_{C}^{+(r)}$.

Conversely, we show that if $\varepsilon=\varepsilon_{0}^{x_{0}} \varepsilon_{1}^{x_{n-1}} \cdots \varepsilon_{n-1}^{x_{1}} \in E_{C}^{+(r)}$, then $\varepsilon_{0}^{x_{0}} \varepsilon_{1}^{x_{1}} \cdots \varepsilon_{n-1}^{x_{n-1}}$ $\in E_{U}^{(r)}$. By above argument it suffices to show that if $\sum_{i=0}^{n-1} x_{i} c_{k-\imath}^{(r)} \equiv 0(\bmod 2)$ for every $k$, then we have

$$
\sum_{i=0}^{n-1} x_{\imath}\left(\frac{-1}{1-\zeta^{2 g^{i}}}+\frac{1}{1-\zeta^{2 g^{i+1}}}\right) \equiv 0(\bmod 2) .
$$

Put $H(i)=\frac{-1}{1-\zeta^{2 g^{i}}}+\frac{1}{1-\zeta^{2 g^{i+1}}}$ and $H_{0}(i)=\frac{-1}{1-\zeta_{0}^{2 g^{i}}}+\frac{1}{1-\zeta_{0}^{2 g^{i+1}}}$ for every $i$.

Then, noting that $H_{0}(i+m)=-H_{0}(i)$ for every $i$, we have

$$
\begin{aligned}
p^{r} \sum_{i=0}^{n-1} x_{i} H(i) & =\sum_{i=0}^{n-1} x_{i} \sum_{k=0}^{p^{r}-1} k\left(\zeta^{2 g^{i} k}-\zeta^{2 g^{i+1} k}\right) \\
& =\sum_{i=0}^{n-1} x_{i} \sum_{\substack{k=0 \\
(k, p)=1}}^{p^{r}-1} k\left(\zeta^{2 g^{i} k}-\zeta^{2 g^{i+1} k}\right)+p \sum_{i=0}^{n-1} x_{i} \sum_{k=0}^{p^{r-1}-1} k\left(\zeta_{0}^{2 g^{i} k}-\zeta_{0}^{2 g^{i+1} k}\right)
\end{aligned}
$$




$$
\begin{aligned}
& =\sum_{i=0}^{n-1} x_{i} \sum_{u=0}^{2 n-1} g_{u}^{(r)}\left(\zeta^{2 g^{i+u}}-\zeta^{2 g^{i+u+1}}\right)+p^{r} \sum_{i=0}^{n-1} x_{i} H_{0}(i) \\
& =\sum_{i=0}^{n-1} x_{i} \sum_{u=0}^{2 n-1}\left(g_{u}^{(r)}-g_{u-1}^{(r)}\right) \zeta^{2 g^{i+u}}+p^{r} \sum_{i=0}^{m-1} \sum_{j=0}^{\frac{n}{m}-1} x_{i+m j} H_{0}(i+m j) \\
& \equiv \sum_{k=n-1}^{3 n-2} \zeta^{2 g^{k}} \sum_{i=0}^{n-1} x_{i} c_{k-i-s-1}^{(r)}+\sum_{i=0}^{m-1} \sum_{j=0}^{\frac{n}{m}-1} x_{i+m j} H_{0}(i) \quad(\bmod 2) \\
& \equiv \sum_{i=0}^{m-1} y_{i} H_{0}(i) \quad(\bmod 2) .
\end{aligned}
$$

Here, by the assumption of induction, we use the following fact: $\eta_{0}^{y_{0}} \eta_{1}^{y_{m-1}} \cdots \eta_{m-1}^{y_{1}}$ $\in E_{C}^{+(r-1)}$ implies $\eta_{0}^{y_{0}} \eta_{1}^{y_{1}} \cdots \eta_{m-1}^{y_{m-1}} \in E_{U}^{(r-1)}$, which is equivalent to $\sum_{i=0}^{m-1} y_{\imath} H_{0}(i) \equiv$ $0(\bmod 2)$. Therefore we obtain

$$
\sum_{i=0}^{n-1} x_{i} H(i)=\sum_{i=0}^{n-1} x_{i}\left(\frac{-1}{1-\zeta^{2 g^{i}}}+\frac{1}{1-\zeta^{2 g^{i+1}}}\right) \equiv 0(\bmod 2) .
$$

Thus the equivalence in Theorem 1 is proved. The characterization of $E_{U}^{(r)}$ is easily deduced by Lemma 1 . This completes the proof of Theorem 1.

Lemma 4. Let $L$ be a real abelian field with conductor $p^{r}$. Then $h_{L}$ is even if and only if $E_{C}^{+} / E_{C}^{2} \cap E_{U} / E_{C}^{2} \neq\{1\}$.

Proof. Suppose that $h_{L}$ is even. Since $h_{L}=\left[E_{L}: E_{C}\right]$, there exists a unit $\varepsilon$ of $E_{L}$ such that $\varepsilon^{2} \in E_{C}$ and $\varepsilon \notin E_{C}$. Therefore $\varepsilon^{2} E_{C}^{2} \neq E_{C}^{2}$ is an element of $E_{C}^{+} / E_{C}^{2}$ $\cap E_{U} / E_{C}^{2}$.

Conversely, if $E_{C}^{+} / E_{C}^{2} \cap E_{U} / E_{C}^{2} \neq\{1\}$, there is a unit $\varepsilon$ of $E_{C}^{+} \cap E_{U}$ which is not contained in $E_{C}^{2}$. Here we may assume that $L(\sqrt{\varepsilon}) / L$ is an extension of degree 2. Because $\sqrt{\varepsilon} \in L$ implies that $h_{L}=\left[E_{L}: E_{C}\right]$ is even. Therefore, since $\varepsilon \in$ $E_{U}$, it follows from Satz 120 in Hecke [9] that any prime ideal of $L$ is unramified in $L(\sqrt{\varepsilon}) / L$. On the other hand $L(\sqrt{\varepsilon}) / L$ is also unramified at all infinite prime divisors of $L$, because $\varepsilon$ is totally positive. This implies that $h_{L}$ is even.

Remark 3. Combining Lemma 4 with Theorem 1, we easily obtain that if $\rho_{L}>[m / 2]$, then $h_{L}$ is even, where [ ] is the Gaussian symbol. However the converse is not valid in general. For example, let $L$ be the real cyclic field of degree 31 with conductor 116933 . Then we have $\mu_{L}=\rho_{L}=10$, so that $2 \mid h_{L}$. On the other hand, if $L$ is the subfield of $\mathbf{Q}\left(\zeta_{311}\right)$ of degree 31 , then $\mu_{L}=0$ and $\rho_{L}=$ 10. So we have $2 \Varangle h_{L}$. Therefore these examples show that the parity of class number of a real abelian field $L$ with prime conductor is not determined by $\rho_{L}$. 
Proof of Corollary. We consider the homomorphism $\phi$ from $E_{C}$ into the set of vectors of size $m$ with components in $\mathbf{F}_{2}$, which is defined by

$$
\eta_{0}^{x_{0}} \eta_{1}^{x_{1}} \cdots \eta_{m-1}^{x_{m-1}} \mapsto^{t}\left(\overline{x_{0}}, \overline{x_{1}}, \cdots, \overline{x_{m-1}}\right),
$$

where $\bar{x}_{\imath}=x_{i}+2 \mathbf{Z}$ for each $i$. Clearly the kernel of $\phi$ is $E_{C}^{2}$. Let $X_{L}=$ $\psi\left(E_{C}^{+}\right)$and $Y_{L}=\phi\left(E_{U}\right)$. Then, by Lemma 1 and Theorem 1 , we have

$$
X_{L}=\left\{x ; M_{L} x=\mathbf{o}\right\} \text { and } Y_{L}=\left\{x ; M_{L}^{*} x=\mathbf{o}\right\},
$$

where $\boldsymbol{x}={ }^{t}\left(\bar{x}_{0}, \bar{x}_{1}, \cdots, \overline{x_{m-1}}\right)$ and $\mathbf{o}$ is the zero vector of size $m$. Hence we obtain

$$
X_{L} \cap Y_{L}=\left\{x ;\left(\begin{array}{c}
M_{L} \\
M_{L}^{*}
\end{array}\right) x=\mathbf{o}\right\},
$$

where $\boldsymbol{o}$ is the zero vector of size $2 m$. So the definition of $\mu_{L}$ shows that \# $\left(X_{L} \cap\right.$ $\left.Y_{L}\right)=2^{\mu_{L}}$. Therefore it follows from Lemma 4 that $h_{L}$ is even if and only if $X_{L} \cap$ $Y_{L} \neq\{\mathbf{o}\}$, i.e., $\mu_{L}>0$. This completes the proof of Corollary of Theorem 1 .

\section{Proof of Theorem 2 and Corollary}

Let $p$ be an odd prime. Let $K$ be an imaginary abelian field with conductor $p^{r}$, i.e., an imaginary subfield of $\mathbf{Q}(\zeta)$ not contained in $\mathbf{Q}\left(\zeta^{p}\right)$, where $\zeta=\zeta_{p}$. Let $h_{K}^{*}$ be the relative class number of $K$. Let $K_{0}$ be the maximal real subfield of $K$. Let $m$ be the degree of $K_{0}$. Regarding $K_{0}$ as $L$, we use the same notations as in section 1 . Let $Q_{K}$ be the unit index of $K$ and $w_{K}$ the number of roots of unity in $K$. The relative class number $h_{K}^{*}$ is given by

$$
h_{K}^{*}=Q_{K} w_{K} \prod_{\chi_{1}} \frac{1}{2 f\left(\chi_{1}\right)} \sum_{a=1}^{f\left(\chi_{1}\right)}-\chi_{1}(a) a,
$$

where $\chi_{1}$ runs through the odd characters of $K$ and $f\left(\chi_{1}\right)$ is the conductor of $\chi_{1}$ (cf. [7]). Here we notice that for any odd character $\chi_{1}$ of $K$

$$
\frac{1}{f\left(\chi_{1}\right)} \sum_{a=1}^{f\left(\chi_{1}\right)} \chi_{1}(a) a=\frac{1}{p^{r}} \sum_{a=1}^{p^{r}} \chi_{1}(a) a .
$$

Therefore, since $Q_{K}=1$ and $w_{K}=2 p^{b}$, where $b=r$ or 0 according as $K=$ $\mathbf{Q}(\zeta)$ or not, we have

$$
h_{K}^{*}=2 p^{b}\left|\prod_{j=0}^{m-1} \frac{1}{2 p^{r}} \sum_{a=1}^{p^{r}-1} \chi^{2 j+1}(a) a\right|,
$$

Here $\chi$ is a generating character of $K$. Put $\alpha=\chi(g)$. Then $\alpha$ is a primitive $2 m$ th 
root of unity. So putting $F(x)=\sum_{i=0}^{2 n-1} g_{i} x^{i}$ where $2 n=\varphi\left(p^{r}\right)$, we obtain

$$
h_{K}^{*}=\frac{2 p^{b}}{\left(2 p^{r}\right)^{m}}\left|F(\alpha) F\left(\alpha^{3}\right) \cdots F\left(\alpha^{2 m-1}\right)\right| .
$$

We put $A_{i}=\sum_{j=0}^{\frac{n}{m}-1} g_{i+2 m j}$ for any $i$. Then, on $\left\{\alpha^{k} \mid k \in \mathbf{Z}\right\}, F(x)=\sum_{i=0}^{2 m-1} A_{\imath} x^{i}$ since $\alpha^{2 m}=1$. Hence, for any odd integer $k$, we have

$$
\begin{aligned}
\left(1-\alpha^{-k}\right) F\left(\alpha^{k}\right) & =\sum_{i=0}^{2 m-1}\left(A_{i}-A_{i+1}\right) \alpha^{i k} \\
& =\sum_{i=0}^{m-1}\left(A_{i}-A_{i+1}\right) \alpha^{i k}+\sum_{i=0}^{m-1}\left(A_{i+m}-A_{i+m+1}\right) \alpha^{(i+m) k} \\
& =2 \sum_{i=0}^{m-1}\left(A_{i}-A_{i+1}\right) \alpha^{i k}
\end{aligned}
$$

because $A_{i}+A_{i+m}=\frac{p^{r} \varphi\left(p^{r}\right)}{2 m}$ for any $i$ and $\alpha^{k m}=-1$ for any odd $k$. It is obvious that $\Pi_{j=0}^{m-1}\left(1-\alpha^{-2 j-1}\right)=2$. Hence, putting $G(x)=\sum_{i=0}^{m-1}\left(A_{i}-A_{i+1}\right) x^{i}$, we have

$$
p^{r m-b} h_{K}^{*}=\left|G(\alpha) G\left(\alpha^{3}\right) \cdots G\left(\alpha^{2 m-1}\right)\right|=\left|\operatorname{det}\left(A_{i+j}-A_{i+j+1}\right)_{0 \leq i, j<m}\right| .
$$

Here, as to the second equality, we refer to the probrem 5 in [2] p. 367. Since $n / m$ is odd, we set $n / m=2 v+1$. Then

$$
\begin{aligned}
A_{i+s}-A_{i+s+1} & =\sum_{j=0}^{2 v}\left(g_{i+s+2 m j}-g_{i+s+1+2 m j}\right) \equiv \sum_{j=0}^{2 v} c_{i+2 m j}(\bmod 2) \\
& =\left\{\sum_{j=0}^{v}+\sum_{j=v+1}^{2 v}\right\} c_{i+2 m j}=\sum_{j=0}^{v} c_{i+2 m j}+\sum_{j=0}^{v-1} c_{i+2 m(j+v+1)} \\
& =\sum_{j=0}^{v} c_{i+2 m j}+\sum_{j=0}^{v-1} c_{i+m+2 m j}=\sum_{j=0}^{2 v} c_{i+m j} .
\end{aligned}
$$

Therefore $A_{i+s}-A_{i+s+1} \equiv d_{i}(\bmod 2)$ for any $i$. Thus we obtain

$$
\begin{aligned}
h_{K}^{*} & \equiv \operatorname{det}\left(A_{i+j}-A_{i+j+1}\right) \equiv \operatorname{det}\left(A_{i+j+s}-A_{i+j+s+1}\right) \\
& \equiv \operatorname{det}\left(d_{i+j}\right)=\operatorname{det} M_{K_{0}}(\bmod 2) .
\end{aligned}
$$

This completes the proof of Theorem 2. Corollary is an immediate consequence of Theorem 2 by the definition of $\rho_{K_{0}}$.

\section{Properties of $\rho_{L}$ and $\mu_{L}$}

In this section we shall give three properties of $\rho_{L}$ and $\mu_{L}$, which are useful to 
calculate $\rho_{K}$ and $\mu_{K}$ for the maximal real subfield $K$ of $\mathbf{Q}\left(\zeta_{p^{r}}\right)$. We note that $\rho_{\mathbf{Q}}=$ $\mu_{\mathbf{Q}}=0$.

Proposition 1. Let $L$ be a real subfield of $\mathbf{Q}\left(\zeta_{p^{r}}\right)$ and $F$ a subfield of $L$. Let $h^{*}$ be the relative class number of $\mathbf{Q}\left(\zeta_{p^{r}}\right)$ and a the integer such that $2^{a} \| h^{*}$. Then $\rho_{F}$ $\leq \rho_{L} \leq$ a. Moreover, if $\rho_{F}=\rho_{L}$, then $\mu_{F}=\mu_{L}$.

Proposition 2. Let $F \subseteq L$ be real subfields of $\mathbf{Q}\left(\zeta_{p^{r}}\right)$. Suppose that $L / F$ is an extension of 2-power degree. Then $\mu_{F}=0$ (resp. $\rho_{F}=0$ ) if and only if $\mu_{L}=0$ (resp. $\left.\rho_{L}=0\right)$.

Proposition 3. Let $F \subseteq L$ be real subfields of $\mathbf{Q}\left(\zeta_{p} r\right)$. Suppose that $L / F$ is an extension of prime degree $l>2$. Let $f$ be the order of 2 modulo $l$. Then $\rho_{L} \equiv \rho_{F}$ and $\mu_{L} \equiv \mu_{F}(\bmod f)$.

We here prove Proposition 1 . Let $K$ be the maximal real subfield of $\mathbf{Q}\left(\zeta_{p} r\right)$. We first show that $\rho_{K} \leq a$. Let $n=\varphi\left(p^{\gamma}\right) / 2$ and $A=\left(g_{i+j}-g_{i+j+1}\right)_{0 \leq \imath, j<n}$. By the proof of Theorem 2 we have $p^{r n-r} h^{*}=|\operatorname{det} A|$. Here we note that $g_{i+s}-$ $g_{i+s+1} \equiv c_{\imath}(\bmod 2)$, where $s$ is the integer such that $g_{s}=2,1 \leq s<\varphi\left(p^{r}\right)$. Therefore the reduction modulo 2 of $M_{K}=\left(c_{1+j}\right)_{0 \leq i, j<n}$ equals $\left(\overline{g_{1+1+s}}-\right.$ $\left.\overline{g_{i+j+s+1}}\right)_{0 \leq i, j<n}$, where $\bar{g}_{i}=g_{1}+2 \mathbf{Z}$ for each $i$. Hence $M_{K}$ and $A$ have the same $\mathbf{F}_{2}$-rank $n-\rho_{K}$. Thus we obtain $2^{\rho_{K}} \mid h^{*}$, which implies $\rho_{K} \leq a$. Next we define $X_{K}$ and $Y_{K}$ for $K$ just as $X_{L}$ and $Y_{L}$ are defined for $L$ in the proof of Corollary of Theorem 1. Let $m=[L: \mathbf{Q}]$. We consider the map $i ; \mathbf{F}_{2}^{m} \rightarrow \mathbf{F}_{2}^{n}$ which is defined by

$$
x \mapsto(x, x, \cdots, x),
$$

where $\mathbf{F}_{2}^{m}$ is the direct sum of $m$ copies of $\mathbf{F}_{2}$ and $\boldsymbol{x}=\left(x_{0}, x_{1}, \cdots, x_{m-1}\right)$. Then $i$ gives natural inclusions $X_{L} \hookrightarrow X_{K}$ and $Y_{L} \hookrightarrow Y_{K}$. Hence we have $\rho_{L} \leq \rho_{K} \leq a$. Similar argument shows that $X_{F} \hookrightarrow X_{L}$ and $Y_{F} \hookrightarrow Y_{L}$. So $\rho_{F} \leq \rho_{L}$. Therefore the assumption $\rho_{F}=\rho_{L}$ implies that $X_{F}=X_{L}$ and $Y_{F}=Y_{L}$. Thus we have $\mu_{F}=\mu_{L}$.

Most part of Proposition 2 is an immediate consequence of Theorems in [10] and our Corollary of Theorem 1. But it is directly proved by using the matrices $M_{L}$ and $M_{L}^{*}$, etc. and by calculating their $\mathbf{F}_{2}$-ranks as follows. Indeed, since 0 $\leq \mu_{F} \leq \mu_{L}$, we may show that $\mu_{F}=0$ implies $\mu_{L}=0$, with assuming $[L: F]=2$. Let $[F: \mathbf{Q}]=m$. Let $d_{i}$ be the integer defined in section 1 , that is, $d_{i}=0$ or 1 according as $\eta_{i}=N_{\mathbf{Q}^{\left(\zeta+\zeta^{-1}\right) / L}}\left(\varepsilon_{\imath}\right)$ is positive or negative. Since $[L: \mathbf{Q}]=2 m$, we 
have $d_{i+2 m}=d_{\imath}$ for every $i$, and $M_{L}=\left(d_{i+j}\right)_{0 \leq i, j<2 m}, M_{L}^{*}=\left(d_{i-j}\right)_{0 \leq i, j<2 m}$. Suppose that $\mu_{F}=0$, i.e., $\operatorname{rank}_{\mathbf{F}_{2}}\left(\begin{array}{c}M_{F} \\ M_{F}^{*}\end{array}\right)=m$. Then there are $2 m$ by $2 m$ matrix $Q$ and $m$ by $m$ matrix $R$ such that

$$
Q\left(\begin{array}{c}
M_{F} \\
M_{F}^{*}
\end{array}\right) R=\left(\begin{array}{c}
E_{m} \\
O
\end{array}\right)
$$

where $E_{m}$ is $m$ by $m$ unit matrix and $O$ is $m$ by $m$ zero matrix. Now, putting $A=$ $\left(d_{i+j}\right)_{0 \leq i, j<m}$ and $B=\left(d_{i+j+m}\right)_{0 \leq i, j<m}$, then

$$
M_{L}=\left(\begin{array}{cc}
A & B \\
B & A
\end{array}\right)
$$

and $M_{F} \equiv A+B(\bmod 2)($ cf. Proof of Lemma 3 in [14]). Then, by definition

$$
M_{L}^{*}=\left(\begin{array}{ll}
A^{*} & B^{*} \\
B^{*} & A^{*}
\end{array}\right)
$$

where $A^{*}=\left(d_{i-j}\right)_{0 \leq i, j<m}$ and $B^{*}=\left(d_{i-j+m}\right)_{0 \leq i, j<m}$. Since $M_{F}^{*} \equiv A^{*}+B^{*}$ $(\bmod 2)$, we have

$$
\begin{aligned}
\operatorname{rank}_{\mathbf{F}_{2}}\left(\begin{array}{c}
M_{L} \\
M_{L}^{*}
\end{array}\right) & =\operatorname{rank}_{\mathbf{F}_{2}}\left(\begin{array}{cc}
A & B \\
B & A \\
A^{*} & B^{*} \\
B^{*} & A^{*}
\end{array}\right)=\operatorname{rank}_{\mathbf{F}_{2}}\left(\begin{array}{cc}
A & B \\
M_{F} & M_{F} \\
A^{*} & B^{*} \\
M_{F}^{*} & M_{F}^{*}
\end{array}\right) \\
& =\operatorname{rank}_{\mathbf{F}_{2}}\left(\begin{array}{cc}
A & M_{F} \\
M_{F} & O \\
A^{*} & M_{F}^{*} \\
M_{F}^{*} & O
\end{array}\right) .
\end{aligned}
$$

Therefore, using above matrices $Q$ and $R$, we obtain

$$
\left(\begin{array}{ll}
Q & O \\
O & Q
\end{array}\right)\left(\begin{array}{cc}
A & M_{F} \\
A^{*} & M_{F}^{*} \\
M_{F} & O \\
M_{F}^{*} & O
\end{array}\right)\left(\begin{array}{cc}
R & O \\
O & R
\end{array}\right)=\left(\begin{array}{cc}
S_{1} & E_{m} \\
S_{2} & O \\
E_{m} & O \\
O & O
\end{array}\right),
$$

where $\left(\begin{array}{c}S_{1} \\ S_{2}\end{array}\right)=Q\left(\begin{array}{c}A \\ A^{*}\end{array}\right) R$. Thus we get $\mu_{L}=0$. Similarly we can show that $\rho_{F}=$ 0 implies $\rho_{L}=0$. This completes the proof of Proposition 2 . 
Proposition 3 is proved as follows. Let $m$ and $n$ be the degrees of $F$ and $L$, respectively. As shown in the proof of Proposition 1, we may regard $X_{F}$ and $Y_{F}$ as subgroups of $X_{L}$ and $Y_{L}$, respectively. Then $G(L / F)$ naturally acts on $X_{L} / X_{F}$, that is, $\left\{\left(x_{0}, x_{1}, \ldots, x_{n-1}\right) X_{F}\right\}^{\sigma}=\left(x_{n-m}, \cdots, x_{n-1}, x_{0}, x_{1}, \cdots, x_{n-m-1}\right) X_{F}$ for any $\left(x_{0}, x_{1}, \cdots, x_{n-1}\right) X_{F} \in X_{L} / X_{F}$ and for a generator $\sigma$ of $G(L / F)$. Since $[L: F]=l$ is an odd prime, it easily follows that the orbit of every element of $X_{L} / X_{F}$ except 1 has $l$ elements. Hence $2^{\rho_{L}-\rho_{F}} \equiv 1(\bmod l)$. Thus we obtain $\rho_{L} \equiv \rho_{F}(\bmod f)$. Similarly applying above argument to $X_{L} \cap Y_{L} / X_{F} \cap Y_{F}$, we get $\mu_{L} \equiv \mu_{F}(\bmod$ $f)$. This completes the proof of Proposition 3.

\section{Numerical example}

In this section we tabulate all the values of odd prime $p<3000$ such that the class number of the maximal real subfield $K$ of $\mathbf{Q}\left(\zeta_{p}\right)$ is even. Since $\rho_{K} \geq \mu_{K} \geq 0$, we may examine the primes $p$ such that $\rho_{K}>0$, i.e., $2 \mid h^{*}$ by Corollary of Theorem 2, where $h^{*}$ is the relative class number of $\mathbf{Q}\left(\zeta_{p}\right)$. For such primes $p$, we calculate the values of $\rho_{K}$ and $\mu_{K}$ by Gaussian elimination method and tabulate them.

In the following table we denote by $a$ the integer such that $2^{a} \| h^{*}$, by $L$ a subfield with $\rho_{L}=\rho_{K}$ and by $m$ the degree of $L$. Here, as the value of $a$, we use the value of $k$ in the Table III in [4].

Table. The values of $\rho_{K}$ and $\mu_{K}$ for the maximal real subfield $K$ of the cyclotomic field with prime conductor $p, 2<p<3000$.

\begin{tabular}{r|rrrrr||r|rrrrr}
\hline \multicolumn{1}{c|}{$p$} & \multicolumn{1}{c}{$a$} & $m$ & $\rho_{L}$ & $\rho_{K}$ & $\mu_{K}$ & \multicolumn{1}{|c|}{$p$} & $a$ & $m$ & $\rho_{L}$ & $\rho_{K}$ & $\mu_{K}$ \\
\hline 29 & 3 & 7 & 3 & 3 & 0 & 463 & 3 & 7 & 3 & 3 & 0 \\
113 & 3 & 7 & 3 & 3 & 0 & 491 & 6 & 7 & 6 & 6 & 6 \\
163 & 2 & 3 & 2 & 2 & 2 & 547 & 2 & 3 & 2 & 2 & 2 \\
197 & 3 & 7 & 3 & 3 & 0 & 607 & 4 & 3 & 2 & 2 & 2 \\
239 & 6 & 7 & 3 & 3 & 0 & 659 & 3 & 7 & 3 & 3 & 0 \\
277 & 4 & 6 & 4 & 4 & 4 & 683 & 5 & 31 & 5 & 5 & 0 \\
311 & 10 & 31 & 10 & 10 & 0 & 701 & 3 & 7 & 3 & 3 & 0 \\
337 & 6 & 21 & 6 & 6 & 0 & 709 & 4 & 6 & 4 & 4 & 4 \\
349 & 4 & 6 & 4 & 4 & 4 & 751 & 4 & 15 & 4 & 4 & 0 \\
373 & 5 & 31 & 5 & 5 & 0 & 827 & 6 & 7 & 6 & 6 & 6 \\
397 & 6 & 6 & 4 & 4 & 4 & 853 & 2 & 3 & 2 & 2 & 2 \\
421 & 4 & 15 & 4 & 4 & 0 & 883 & 6 & 63 & 6 & 6 & 0 \\
\hline
\end{tabular}




\begin{tabular}{|c|c|c|c|c|c|c|c|c|c|c|c|}
\hline$p$ & $a$ & $m$ & $\rho_{L}$ & $\rho_{K}$ & $\mu_{K}$ & $p$ & $a$ & $m$ & $\rho_{L}$ & $\rho_{K}$ & $\mu_{K}$ \\
\hline 937 & 2 & 3 & 2 & 2 & 2 & 1879 & 2 & 3 & 2 & 2 & 2 \\
\hline 941 & 8 & 10 & 8 & 8 & 8 & 1951 & 2 & 3 & 2 & 2 & 2 \\
\hline 953 & 3 & 7 & 3 & 3 & 0 & 2011 & 4 & 15 & 4 & 4 & 0 \\
\hline 967 & 3 & 7 & 3 & 3 & 0 & 2131 & 2 & 3 & 2 & 2 & 2 \\
\hline 1009 & 8 & 63 & 8 & 8 & 2 & 2143 & 3 & 7 & 3 & 3 & 0 \\
\hline 1021 & 8 & 255 & 8 & 8 & 0 & 2161 & 4 & 15 & 4 & 4 & 4 \\
\hline 1051 & 6 & 21 & 6 & 6 & 0 & 2221 & 4 & 15 & 4 & 4 & 0 \\
\hline 1093 & 3 & 7 & 3 & 3 & 0 & 2297 & 3 & 7 & 3 & 3 & 0 \\
\hline 1117 & 5 & 31 & 5 & 5 & 0 & 2311 & 5 & 21 & 5 & 5 & 2 \\
\hline 1163 & 3 & 7 & 3 & 3 & 0 & 2381 & 6 & 14 & 6 & 6 & 0 \\
\hline 1171 & 4 & 15 & 4 & 4 & 0 & 2521 & 3 & 7 & 3 & 3 & 0 \\
\hline 1399 & 4 & 3 & 2 & 2 & 2 & 2591 & 3 & 7 & 3 & 3 & 0 \\
\hline 1429 & 3 & 7 & 3 & 3 & 0 & 2689 & 2 & 3 & 2 & 2 & 2 \\
\hline 1471 & 3 & 7 & 3 & 3 & 0 & 2797 & 4 & 6 & 4 & 4 & 4 \\
\hline 1499 & 3 & 7 & 3 & 3 & 0 & 2803 & 2 & 3 & 2 & 2 & 2 \\
\hline 1699 & 2 & 3 & 2 & 2 & 2 & 2843 & 3 & 7 & 3 & 3 & 0 \\
\hline 1777 & 4 & 6 & 4 & 4 & 4 & 2857 & 3 & 7 & 3 & 3 & 0 \\
\hline 1789 & 4 & 6 & 4 & 4 & 4 & 2927 & 6 & 7 & 6 & 6 & 6 \\
\hline
\end{tabular}

\section{REFERENCES}

(1) N. Ankeny, S. Chowla and H. Hasse, On the class number of the maximal real subfield of a cyclotomic field, J. reine angew. Math., 217 (1965), 217-220.

[2] Z. I. Borevich and I. R. Shafarevich, Number Theory, Academic Press, 1966.

[3] G. Cornell and M. I. Rosen, The $l$-rank of the real class group of cyclotomic fields, Compositio Math., 53 (1984), 133-141.

[4] G. Fung, A. Granville and H. C. Williams, Computation of the first factor of the class number of cyclotomic fields, J. Number Theory, 42 (1992), 297-312.

[5] G. Gras, Critère de parité du nombre de classes des extensions abéliennes réelles de $\mathbf{Q}$ de degré impair, Bull. Soc. Math. France, 103 (1975), 177-190.

[6] G. Gras and M.-N. Gras, Signature des unités cyclotomiques et parité du nombre de classes des extensions cycliques de $\mathbf{Q}$ de degré premier impair, Ann. Inst. Fourier, Grenoble, 25 (1975), 1-22.

[7] H. Hasse, Über die Klassenzahl abelscher Zahlkörper, Akademie-Verlag, Berlin, 1952; Springer-Verlag, 1985.

[8] F. Hazama, Demjanenko matrix, class number, and Hodge group, J. Number Theory, 34 (1990), 174-177.

[9] E. Hecke, Vorlesungen über die Theorie der algebraischen Zahlen, Chelsea Pub. Co., 1948.

[10] K. Iwasawa, A note on class numbers of algebraic number fields, Abh. Math. Sem. 
Univ. Hamburg, 20 (1956), 257-258.

[11] E. E. Kummer, Über eine Eigenschaft der Einheiten der aus den Wurzeln der Gleichung $\alpha^{\lambda}=1$ gebildeten complexen Zahlen, und über den zweiten Factor der Klassenzahl, Monatsber. Akad. Wiss., Berlin (1870), 855-880. Reprinted in Collected Papers, vol. I, Springer-Verlag, 1975, 919-944.

[12] W. Schwarz, Demjanenko matrix and 2-divisibility of class numbers, Arch. Math., 60 (1993), 154-156.

[13] L. C. Washington, Introduction to cyclotomic fields, Springer-Verlag, 1982.

[14] K. Yoshino, On the class number of an abelian field with prime conductor, Proc. Japan Acad., 69 A (1993), 278-281.

Department of Mathematics

Kanazawa Medical University

Uchinada-machi, Ishikawa 920-02

Japan 